\title{
Should we afford implantable cardioverter defibrillator therapy?
}

\author{
A John Camm
}

Implantable cardioverter defibrillators (ICDs) quickly earned the image of incontestable life preservation. This view prevented appropriate efficacy trials because the sacrifice for patients not randomized to ICD therapy was deemed too great. A need for clinical trials to properly assess the cost and efficacy of device therapy was, however, argued. The cost of ICDs was notable and some serious adverse events were associated with their use. Trials were not, though, favored by the device companies because of sparse experience with ICDs and the learning curves of the investigators possibly being disadvantageous. It took almost a decade to persuade the industry, health-care payers and doctors that it was right and timely to pursue clinical trials.

So far, only three so-called secondary prevention trials have been done: the Antiarrhythmics Versus Implantable Device (AVID) trial, the Cardiac Arrest Study Hamburg (CASH) and the Canadian Implantable Defibrillator Study (CIDS). Only AVID showed unequivocally that device therapy reduced mortality in survivors of life-threatening ventricular arrhythmias. A meta-analysis of all three studies supported the efficacy of ICDs but suggested much higher costs than previously thought. Nevertheless, ICDs saved lives, and the payers agreed to the costs for patients at high risk of sudden cardiac death.

Attention turned to the use of ICDs in lowerrisk patients. In a small study in patients with left-ventricular ejection fraction (LVEF) lower than $35 \%$ and inducible and nonsuppressible sustained ventricular tachyarrhythmias, optimum therapy plus ICD use compared with medical therapy led to substantial life-saving benefit. A subsequent trial in patients with LVEF lower than $40 \%$ strongly supported the results and suggested that differentiation of post-Ml patients with poor ventricular function was unnecessary to see a benefit. Therefore,
Many

considerations

could make it

easy to restrict

the use of ICDs

AJ Camm is the

Head of Department,

Department of

Cardiac and Vascular

Sciences, St George's

Hospital Medical

School, London, UK.

Competing interests

The author declared

competing interests; go to

the article online for details.

www.nature.com/clinicalpractice doi:10.1038ncpcardio0122 a substantially wider population of patients would benefit from ICD therapy, but could this approach really be affordable?

Further trials have better defined the risk among post-Ml patients. Sudden cardiac death can be prevented in patients with very poor ventricular function $(<30 \%)$ and possibly those with equally poor ventricular function due to nonischemic causes (dilated cardiomyopathy). ICDs also save lives among NYHA class II and III heart failure patients with poor ventricular function (LVEF <35\%) but no known ventricular arrhythmia, especially class II patients, irrespective of the cause of ventricular dysfunction. Since the absolute risk of mortality in this population is, however, relatively small, so is the absolute risk reduction.

The combination of a large target population but reduced absolute benefit has put ICD therapy back on to the affordability agenda of health payers throughout Europe and North America. There are several approaches to deal with cost-effectiveness issues: denial of therapy to all patients on the grounds of expense; unrestricted use of ICDs; the writing of guidelines taking in account the available evidence to encourage health-care payers to decide the strength of recommendation they are prepared to afford; or better definition of the eligibility of patients. Of these options the latter seems most likely to succeed. Since further randomized clinical trials are generally not feasible, large databases of ICD recipients should be urgently instituted to allow improved risk stratification.

Many considerations could make it easy to restrict the use of ICDs. It is not the role of the medical profession, however, to collude with attempts to impede the proper use of life-saving therapy. That everything must be done to preserve life is a code to which physicians have adhered for centuries and must not now relinquish. 